\title{
Comparison of Two surgical treatments for Rhegmatogenous Retinal Detachment
}

\section{Tal Yahalomi *, Idan Hecht ${ }^{2,3}$, Michal Lifshitz ${ }^{1}$, Roee Arnon ${ }^{1}$, Halit Winter $^{1}$ and Joseph Pikkel ${ }^{1}$}

${ }^{1}$ Department of Ophthalmology, Samson Assuta Ashdod Hospital, Faculty of Health

Sciences, Ben-Gurion University of the Negev, Israel

${ }^{2}$ Sackler Faculty of Medicine, Tel Aviv University, Tel Aviv, Israel

${ }^{3}$ Department of Ophthalmology, Shamir Medical, Zerifin, Israel

*Corresponding Author: Tal Yahalomi, Department of Ophthalmology, Samson Assuta Ashdod Hospital, Faculty of Health Sciences, Ben-Gurion University of the Negev, Israel.
Received: November 22, 2021

Published: December 15, 2021

(C) All rights are reserved by Tal Yahalomi., et al.

\begin{abstract}
Purpose: To compare the success rate of pars plana vitrectomy (PPV) and scleral buckling in terms of visual outcomes, repeated surgeries and postoperative complications for the treatment of rhegmatogenous retinal detachment.

Methods: We collected data of all patients operated in one tertiary medical center in Israel during the year 2019. Patient over the age of 18 years with at least one year of follow-up were included. Retinal attachment rate, visual outcomes and postoperative complications were compared.

Results: Included were 30 patients that underwent posterior vitrectomy and 36 that underwent scleral buckling. All patients were operated by the same surgeon. Success rate of retina re-attachment were almost the same in both groups $(86.7 \%$ vs 86.1 for vitrectomy and scleral buckle respectively, $p=0.948$ ). In both groups there was a significant improvement of the final visual acuity compared to the pre-operative visual acuity $(0.146 \pm 0.1$ vs $0.149 \pm 0.1$ Log MAR improvement for vitrectomy and scleral buckle respectively, $\mathrm{p}$ $=0.411$ ). A strong correlation existed between the number of operations to reattachments -any additional surgery drops the chance of reattachment by $2.4 \%$ (odds ratio: $0.024,95 \%$ CI $0.003-0.208, \mathrm{p} \leq 0.001$ ).

Conclusion: Among a modern cohort of patients with retinal detachment, no differences were seen in success rates or visual outcomes between patients treated with posterior vitrectomies compared to scleral buckling.
\end{abstract}

Keywords: Pars Plana Vitrectomy; PPV; Scleral Buckling; SB; Rhegmatogenous Retinal Detachment; RRD

\section{Introduction}

The etiology behinds rhegmatogenous retinal detachment is the accumulation of fluid behind the sensorineural retina following a retinal tear, hole or flap [1]. If symptomatic rhegmatogenous retinal detachment had occurred, most patients will become blind unless they undergo surgical repair [2]. There are several ways to reattach the retina following rhegmatogenous retinal detachment, two of the predominant methods are posterior trans pars plana vitrectomy (PPV) and scleral buckling (SB).

The main idea behind SB is by preventing passage of fluid from the vitreous body and allowing retinal re-adherence [3]. In addi- tion, the pressure prevents leak of fluid from the vitreous body to the sub-retinal space [4]. The idea behind posterior vitrectomy is resection of the vitreous body and areas stretching the retina. After the resection gas or silicone oil tamponade is performed to flatten the retina and give time to natural adherence process of the tear area [3].

Most studies comparing the two methods date almost 20 years. A 2019 Cochrane review by Znaor., et al. included 10 trials totaling 1307 eyes dating from 2002-2007 [2]. They found no difference between PPV and scleral buckling in terms of success rate and vision achieved. Since then, however, PPV has gained in popularity 
for many surgeons, particularly for patients with pseudophakic RRD, thanks to recent advances in vitrectomy techniques. These advancements in devices and techniques, together with the increased experience of surgeons and faster surgical time, could have significant influence the outcomes of the surgery and the rate of complications. These advances call for a modern comparison between methods. Relatively little evidence exists comparing these two techniques using modern methods and equipment, and their results are conflicting [5].

Each of these methods have its' advantages and disadvantages. While some surgeons decide whether to choose one procedure counting on patients age, retinal tear location etc. others still are confused and usually make their decision based on personal experience or on personal confidence.

In this study we compared PPV and SB in terms of efficacy and safety among patients with rhegmatogenous retinal detachment operated on in single tertiary center in 2019.

\section{Materials and Methods}

This study adhered to the tenets of the Declaration of Helsinki and was approved by the institutional review board (IRB) of the Samson Assuta Ashdod Hospital. Due to its retrospective nature, a waiver of informed consent was granted.

We collected the data of all patients operated in one tertiary center in Israel (Samson Assuta Ashdod Hospital) during the year 2019. Excluded were patients that did not have follow up data if at least one year and patients that were under the age of 18 years. Demographic data as well as all pre and post operation data were collected. Myopia was considered above 2 Diopter.

The main outcome was rate of retina re-attachment one month from surgery. Secondary outcomes were improvement of the final visual acuity, rates of re-operations needed and rate of intra- and post-operative complications.

\section{Surgical Procedures}

Patients underwent 23-gauge pars plana vitrectomy with injection of perfluorocarbon liquid (PFCL). All retinal tears were circuled with endolaser, and PFCL was replaced by SF6 gas. After surgery all patients were positioned with face downward for 6 hours during hospitalization and were guided with subsequent position until follow-up according to the location of the retinal tears. All surgeries were performed by one experienced vitreoretinal surgeon (JP).

\section{Statistical evaluation}

For continuous variables summary tables are provided giving arithmetic mean (M) and standard deviation (Sd). For categorical variables summary table is provided giving sample size, absolute and relative frequencies. Pearson Chi-square tests were applied for testing the correlations between the study groups for the categorical parameters. While for quantitative parameters, independent sample t-tests were applied for testing differences between study groups, and Pearson correlations were applied to test the power of the correlations. All tests applied are two-tailed, and p-value of 5\% or less was considered statistically significant. The data was analyzed using the SPSS version 20.0.0.2 (SPSS Inc. Chicago, IL, USA).

\section{Results}

Included were 30 patients that underwent PPV and 36 patients that underwent SB. All patients were operated by the same surgeon (J.P.).

The mean age was 54.6 years and 44 were male. 33 were pseudophakic and 31 were myopic. There were no significant differences between the groups in baseline demographic information (Table 1), including age (55.1 vs $54.6, \mathrm{p}=0.826)$, gender (19 (63.3\%) vs. $25(69.4 \%)$ males in each group, $p=0.600)$, baseline visual acuity ( 0.216 vs. $0.218, p=0.960$ ), nor in the rate of maculae on vs maculae off at admission (12 vs 15 presented with macula off, $p=0.89$ ), 13 patients underwent silicon oil injection in the PPV group.

\begin{tabular}{|l|c|c|c|c|c|}
\hline & \multicolumn{2}{|c|}{$\begin{array}{c}\text { Posterior } \\
\text { Vitrectomy } \\
\text { (n = 30) }\end{array}$} & \multicolumn{2}{c|}{$\begin{array}{c}\text { Scleral } \\
\text { Buckling } \\
\text { (n = 36) }\end{array}$} & \\
\hline & $\mathrm{M}$ & $\mathrm{Sd}$ & $\mathrm{M}$ & $\mathrm{Sd}$ & $\mathrm{p}$ \\
\hline Age (years) & 55.1 & 10.1 & 54.6 & 10.0 & 0.826 \\
\hline Surgeries (n) & 1.4 & 0.8 & 1.4 & 0.7 & 0.697 \\
\hline Visual acuity (before) & 0.216 & 0.163 & 0.218 & 0.167 & 0.960 \\
\hline Visual acuity (after) & 0.392 & 0.235 & 0.367 & 0.214 & 0.653 \\
\hline$\Delta$ visual acuity & 0.146 & 0.142 & 0.149 & 0.123 & 0.411 \\
\hline & $\mathrm{N}$ & $\%$ & $\mathrm{~N}$ & $\%$ & \\
\hline Gender (Male) & 19 & 63.3 & 25 & 69.4 & 0.600 \\
\hline Use of silicon (yes) & 13 & 43.3 & - & - & - \\
\hline Myopia (yes) & 11 & 36.7 & 20 & 55.6 & 0.126 \\
\hline Macula (off) & 12 & 40.0 & 15 & 41.5 & 0.891 \\
\hline Pseudophakia (yes) & 15 & 50.0 & 18 & 50.0 & 0.999 \\
\hline Cataract (yes) & 8 & 26.7 & 7 & 19.4 & 0.486 \\
\hline Intraocular Pressure & 10 & 33.3 & 13 & 36.1 & 0.814 \\
(yes) & 26 & 86.7 & 31 & 86.1 & 0.948 \\
\hline Re-attachment & & & & & \\
\hline
\end{tabular}

Table 1: Demographic characteristics of the study participants according to the type of surgery. 
For the primary endpoint of retina re-attachment results were similar between groups. 26 (86\%) vs 31 (86\%), $p=0.948)$. In both groups there was a significant improvement of the final visual acuity compared to the pre-operative visual acuity $(\mathrm{r}=-0.808, \mathrm{p}<$ 0.001 ), however comparing final visual acuity both groups showed similar improvement ( 0.392 vs. $0.367, \mathrm{p}=0.65)$, and the delta in visual acuity following treatment showed in both groups the same improvement $((0.146 \pm 0.1$ vs $0.149 \pm 0.1$ Log MAR improvement for vitrectomy and scleral buckle respectively, $\mathrm{p}=0.411$ ).

Out of 13 patients that were treated with intra-vitreal silicon oil, four had an elevated intra-ocular pressure however comparing the two groups there was not statistically difference in the number of patients with post-operative intra ocular pressure elevation (10 vs $13, \mathrm{p}=0.814)$.

In predicting success of reattachment - there was a strong correlation between the number of operations to reattachments-the more procedures the less success-any additional surgery drops the chance of reattachment by 0.024 (Table 2).

\begin{tabular}{|l|c|c|c|c|c|}
\hline & B & S.E. & $\begin{array}{c}\text { Wald } \\
(\mathbf{d f}=\mathbf{1})\end{array}$ & Exp(B) & $\mathbf{9 5 \% ~ C I ~}$ \\
\hline $\begin{array}{l}\text { Number of } \\
\text { surgeries }\end{array}$ & -3.75 & 1.10 & $11.55^{* * *}$ & 0.024 & $0.003-0.208$ \\
\hline
\end{tabular}

Table 2: Logistic Regression to predict reattachment by number of surgeries.

Note: $\mathrm{R}^{2}=.69$, Model $\chi_{(1)}^{2}=36.56, \mathrm{p}<.001,{ }^{* * *} \mathrm{p} \leq 0.001$.

\section{Discussion and Conclusion}

In this study we compared PPV to SB for the treatment of retinal detachment. Results show similar rates of retinal re-attachment between groups, similar improvement in visual acuity, and no significant difference in the rate of complications.

RRD occurs in about 0.5 - 1.5 percent of cases one year following cataract surgery, and 30 - 40 percent of patients with RRD are pseudophakic [6-9]. This rate is expected to rise in tandem with the incidence of cataract surgery and the average life expectancy.

PPV has become the first choice of many surgeons, particularly in patients with pseudophakic RRD, thanks to recent advances in vitrectomy techniques, the visualization of small retinal tears, the option of subretinal fluid drainage and retinopexy. Furthermore,
PVR is less likely to occur after this treatment $[10,11]$. The number of retina specialists who favor treating RD with vitrectomy without SB has risen between 2005 and 2015, according to the findings of the Preferences and Trends (PAT) survey done in 2013 [12]. In a survey published in 1997 by Benson., et al. 62 percent of vitreoretinal surgeons favored SB surgery while only 7\% preferred PPV [13]. Since of advances in vitrectomy procedures, as well as the fact that peripheral retinal tears can be properly detected and the prevalence of PVR has decreased, the popularity of PPV in pseudophakic RD surgery has grown over time.

Cankurtaran., et al. retrospectively compared between pseudophakic rhegmatogenous retinal detachment (RRD) patients who underwent PPV and those who underwent SB surgery and revealed no major difference between the primary and final anatomical and visual success rates, nor the rate of retinal detachment recurrence [5]. On the contrary, a retrospective cohort of 822 patients, the PPV group had a higher average proportion of surgical failures than the SB group [14]. In eyes with macula-off RRD treated with PPV, the macular ganglion cell layer-inner plexiform layer (GCL-IPL) was found to be significantly thinner compared to eyes undergoing scleral buckling surgery [15]. Furthermore, Znaor reported in a cocharne review of 10 Randomized controlled trials of 1307 eyes, found no difference between PPV and scleral buckling in terms of operation success and vision achieved and PPV can be linked to a lower risk of detachment recurrence. Few negative outcomes, such as cataract development and new iatrogenic splits, appeared to be more common in those underwent PPV, whereas choroidal detachment appeared to be more common in the scleral buckling group [2]. In an european multicenter, prospective, randomized clinical trial of 416 phakic and 265 pseudophakic eyes with 'medium intensity' RD, postoperative visual acuity was slightly improved in phakic patients treated with SB, whereas in pseudophakic patients treated with PPV, the success rate was significantly higher [16]. Moreover, Brazitikos., et al. found no substantial difference between the two approaches in terms of final visual acuity [17]. Though in each of these procedures are advantages and disadvantages, there are sometimes doubts and hesitations regarding which of these procedures is better. Scleral buckling - though being historically prior to posterior vitrectomy - is considered a more difficult procedure that requires an accurate localization of the retinal tear and results in a refractive error due to elongation of the eyes axial length and sometimes causes significant astigmatism. One 
of two basic methods in the surgical treatment of RD is SB, which provides target-oriented retinal attachment. Before the advances in PPV technique, SB had the highest success rate and was the most used approach in the treatment of RD [18-20]. In patients with aphakic and pseudophakic retinal detachment, the anatomical success rate of SB ranges between $60 \%$ to $80 \%[19,20]$. Small retinal tears that cannot be seen, retinal tears that are located anteriorly, and the occurrence of proliferative vitreoretinopathy (PVR) are the most common causes of ineffective SB [21].

On the other hand - PPV often induces cataract, a significant disadvantage mainly in younger patients that must have a second procedure of cataract extraction and to lose their accommodation ability. The most important benefit of PPV is that it allows for better visualization of peripheral vitreous detachment by removing the vitreous and posterior hyaloid membrane. It also allows microscopic visualization of the peripheral fundus using scleral indentation and internal illumination when used in conjunction with wide-angle imaging systems. As a result, it offers a high rate of anatomical success in treating RD by allowing for better visualization of retinal tears in the peripheral fundus and prompt intervention.

Four of the 13 patients treated with intra-vitreal silicon oil had an increased intra-ocular pressure, but there was no statistical discrepancy between groups in terms of the percentage of patients with post-operative intra-ocular pressure escalation (Table 1). Thus, we have not found any difference between SB and PPV with post-operative intra-ocular pressure escalation.

We tried to tackle the question a bit differently while checking the final visual acuity in both procedures and by trying to see if we can predict reattachment success.

In this study we compared PPV to SB Among a modern cohort of patients with retinal detachment. The results show no differences in success rates or visual outcomes.

\section{Ethics Approval and Consent to Participate}

This study adhered to the tenets of the Declaration of Helsinki and was approved by the institutional review board (IRB) of the Samson Assuta Ashdod Hospital. Due to its retrospective nature, a waiver of informed consent was granted.

\section{Consent for Publication}

Not applicable.

\section{Availability of Data and Materials}

Not applicable.

\section{Competing Interests}

The following authors have no financial disclosures: TY, IH, ML, RA, HW, JP.

\section{Funding}

No funding or grant support.

\section{Authors' Contributions}

All authors attest that they meet the current ICMJE criteria for Authorship.

\section{Acknowledgements}

None.

\section{Bibliography}

1. Dan L Longo. "Harrison's Principles of Internal Medicine”. 18e, chapter 28 ed (2021).

2. Znaor L., et al. "Pars plana vitrectomy versus scleral buckling for repairing simple rhegmatogenous retinal detachments". Cochrane Database Systematic Reviews 2019.3 (2019).

3. Tasman W and Jaeger E. "Duane's Foundations of Clinical Ophthalmology". 2006, chapter 27. Lippincott Williams and Wilkins (2006).

4. Myron Yanoff M., et al. "Ophthalmology". $5^{\text {th }}$ edition (2018).

5. Cankurtaran V., et al. "Anatomical and functional outcomes of scleral buckling versus primary vitrectomy in pseudophakic retinal detachment". Bosnian Journal of Basic Medical Sciences 17.1 (2017): 74-80.

6. Ah-Fat FG., et al. "Trends in vitreoretinal surgery at a tertiary referral centre: 1987 to 1996". British Journal of Ophthalmology 83.4 (1999): 396-398.

7. Olsen G and Olson RJ. "Update on a long-term, prospective study of capsulotomy and retinal detachment rates after cataract surgery". Journal of Cataract and Refractive Surgery 26.7 (2009): 1017-1021.

8. Javitt JC., et al. "National Outcomes of Cataract Extraction I: Retinal Detachment after Inpatient Surgery". Ophthalmology 98.6 (1999): 895-902.

9. Lois N and Wong D. "Pseudophakic retinal detachment". Survey of Ophthalmology 48.5 (2003): 467-487.

10. Speicher MA., et al. "Primary Vitrectomy Alone for Repair of Retinal Detachments Following Cataract Surgery". Retina 20.5 (2000): 459. 
11. Bartz-Schmidt KU., et al. "Primary vitrectomy for pseudophakic retinal detachment". British Journal of Ophthalmology 80.4 (1996): 346-349.

12. Stone TW and Mittra RA. "ASRS 2013 Preferences and Trends Membership Survey, Chicago, IL, USA". American Society of Retina Specialists (2013).

13. W E Benson., et al. "Current popularity of pneumatic retinopexy-PubMed”. Retina (1999): 238-241.

14. Kawano S., et al. "Scleral buckling versus pars plana vitrectomy in simple phakic macula-on retinal detachment: A propensity score-matched, registry-based study". British Journal of Ophthalmology (2021).

15. Gharbiya M., et al. "Macular Ganglion Cell Layer Thickness after Macula-Off Rhegmatogenous Retinal Detachment Repair: Scleral Buckling versus Pars Plana Vitrectomy". Journal of Clinical Medicine 9.5 (2000): 1411.

16. Heimann H., et al. "Scleral Buckling versus Primary Vitrectomy in Rhegmatogenous Retinal Detachment. A Prospective Randomized Multicenter Clinical Study". Ophthalmology 114.12 (2007).

17. Brazitikos PD., et al. "Primary pars plana vitrectomy versus scleral buckle surgery for the treatment of pseudophakic retinal detachment: A randomized clinical trial". Retina 25.8 (2005): 957-964.

18. S Lake., et al. "Management of pseudophakic retinal detachment with various intraocular lens types-PubMed". Annals of Ophthalmology (1993): 381-384.

19. Yoshida A., et al. "Retinal Detachment after Cataract Surgery: Surgical Results". Ophthalmology 99.3 (1992): 460-465.

20. Cousins S., et al. "Pseudophakic Retinal Detachments in the Presence of Various IOL Types". Ophthalmology 93.9 (1986): 1198-1208.

21. Yoshida A., et al. "Retinal Detachment after Cataract Surgery: Predisposing Factors”. Ophthalmology 99.3 (1999): 453-459.

\section{Assets from publication with us}

- Prompt Acknowledgement after receiving the article

- Thorough Double blinded peer review

- Rapid Publication

- Issue of Publication Certificate

- High visibility of your Published work

Website: www.actascientific.com/

Submit Article: www.actascientific.com/submission.php

Email us: editor@actascientific.com

Contact us: +919182824667

Citation: Tal Yahalomi., et al. "Comparison of Two surgical treatments for Rhegmatogenous Retinal Detachment". Acta Scientific Ophthalmology 5.1 (2022): 29-33. 